\title{
Transcendence of infinite sums of simple functions
}

\author{
by \\ Chester Weatherby (Kingston, ON)
}

1. Introduction. In 1969, during a conference on number theory at Stony Brook, S. Chowla asked (see also [5]) if there was a rational-valued periodic function, not identically zero, with prime period $p$ such that the series

$$
\sum_{n=1}^{\infty} \frac{f(n)}{n}
$$

converges and vanishes. In 1973, Baker, Birch and Wirsing [3] answered this question negatively by proving the following theorem:

THEOREM 1. If $f$ is a nonvanishing function defined on the integers with algebraic values and period $q$ such that (i) $f(r)=0$ if $1<(r, q)<q$, (ii) the qth cyclotomic polynomial is irreducible over $\mathbb{Q}(f(1), \ldots, f(q))$, then

$$
\sum_{n=1}^{\infty} \frac{f(n)}{n} \neq 0 .
$$

In 2001, Adhikari, Saradha, Shorey, Tijdeman [1] and then in 2007, Murty and Saradha 10 showed using different methods that when the sum converges it is in fact transcendental. This was done by relating the sum to a linear form in logarithms. In [10 and 1, the authors also examined series of the form

$$
\sum_{n=0}^{\infty} \frac{A(n)}{B(n)} \text { and also } \sum_{n=0}^{\infty} \frac{f(n)}{B(n)}
$$

where $A(x)$ and $B(x)$ are polynomials with rational coefficients, $B(x)$ having only simple rational roots. More recently, the authors of [6] examine similar series, introducing cases where $B(x)$ does not have simple roots. We give our own proofs for various results and extend some results further. After

2010 Mathematics Subject Classification: Primary 11J81; Secondary 11J86, 65B10. Key words and phrases: transcendence, digamma function, infinite series. 
developing a general theory, we show some cases where series of the form

$$
\sum_{n=0}^{\infty} \frac{f(n) A(n)}{B(n)}
$$

are transcendental. In particular, we prove the following theorem.

Theorem 10. Let $A(x), B(x) \in \overline{\mathbb{Q}}[x]$ with $\operatorname{deg}(A)<\operatorname{deg}(B)-1$ be such that $B(x)$ has only simple roots $-p_{1} / q_{1}, \ldots,-p_{k} / q_{k} \in \mathbb{Q}$ with $\left(p_{i}, q_{i}\right)=1$. If there is a $q_{j}>1$ which is coprime to each of the other $q_{i}$ 's, then the sum

$$
\sum_{n=0}^{\infty} \frac{A(n)}{B(n)}
$$

is transcendental.

We write $\sum^{\prime}$ to represent summation which avoids the zeroes of $B(x)$.

The authors of [1] also examine series of the form

$$
\sum_{n=0}^{\infty} \frac{z^{n} A(n)}{B(n)}
$$

where $A(x), B(x)$ are polynomials with algebraic coefficients such that $B(x)$ has simple rational roots, and $z$ is algebraic. We take a different approach which seems to simplify the proofs of some known results. In particular, we prove:

Theorem 17. Let $z \neq 1$ be algebraic with $|z| \leq 1$. Let $A(x), B(x) \in \overline{\mathbb{Q}}[x]$ be such that $B(x)$ has simple rational roots. If it converges, the series

$$
\sum_{n=0}^{\infty} \frac{z^{n} A(n)}{B(n)}
$$

is a computable algebraic number or is transcendental.

2. Preliminaries. We require some knowledge of the digamma function, $\psi(x)$, which is the logarithmic derivative of the gamma function,

$$
\psi(x)=\frac{\Gamma^{\prime}(x)}{\Gamma(x)} .
$$

We do not study the digamma function here, but make use of some well known equalities. By logarithmically differentiating

$$
\Gamma(x+1)=x \Gamma(x)
$$

and the Hadamard product

$$
\frac{1}{\Gamma(x)}=x e^{\gamma x} \prod_{n=1}^{\infty}(1+x / n) e^{-x / n},
$$


we obtain

$$
\psi(x+1)=\psi(x)+\frac{1}{x}
$$

and

$$
-\psi(x)-\gamma=\frac{1}{x}+\sum_{n=1}^{\infty}\left(\frac{1}{n+x}-\frac{1}{n}\right)
$$

where $\gamma$ is Euler's constant. We also recall Lemma 21 from [10], which states that for any integers $1 \leq a \leq q$,

$$
-\psi(a / q)-\gamma=\log q-\sum_{b=1}^{q-1} \zeta_{q}^{-b a} \log \left(1-\zeta_{q}^{b}\right),
$$

where $\zeta_{q}=e^{2 \pi i / q}$ is a primitive $q$ th root of unity. As we see in (3), we can relate special values of the digamma function to logarithms of algebraic numbers. This brings us to Baker's theorem.

Theorem 2 (A. Baker, [2]). For $\alpha_{1}, \ldots, \alpha_{n} \in \overline{\mathbb{Q}} \backslash\{0\}$, if $\log \alpha_{1}, \ldots$, $\log \alpha_{n}$ are linearly independent over $\mathbb{Q}$, then $1, \log \alpha_{1}, \ldots, \log \alpha_{n}$ are linearly independent over $\overline{\mathbb{Q}}$.

Note that throughout this work we take the principal value of the logarithm with argument in $(-\pi, \pi]$. It is easy to see that Theorem 2 implies that any linear combination of logarithms of algebraic numbers with algebraic coefficients is either zero or transcendental. The authors of [10] showed that quantity (3) is nonzero, which, among other implications, yields the following:

Theorem 3 (P. Bundschuh [4, M. R. Murty/N. Saradha [10]). Let $q>1$. Then $\psi(a / q)+\gamma$ is transcendental for any $1 \leq a<q$.

We now define the so-called polygamma functions. The polygamma function $\psi_{k}(x)$ is defined as the $k$ th derivative of the digamma function with $\psi(x)=\psi_{0}(x)$. Differentiating (2) consecutively $k-1$ times we obtain

$$
\psi_{k-1}(x)=(-1)^{k}(k-1) ! \sum_{n=0}^{\infty} \frac{1}{(n+x)^{k}} .
$$

Note that for the last equality we have made the assumption that $x$ is a real variable that is not a negative integer. Equation (4) shows a connection between the polygamma function and the Hurwitz zeta function, which we assume the reader is familiar with. See [10] for a sufficient introduction to the Hurwitz zeta function.

In the sections ahead we will use these tools to say something about infinite sums of simple functions. 
3. Special values of the digamma function. We relate various series to special values of the digamma function. We have all the necessary tools to prove a result similar to Theorem 16 in [10].

THEOREM 4. Let $f$ be a periodic function with period $q \geq 1$ such that

$$
\sum_{a=0}^{q-1} f(a)=0
$$

and take $\alpha>0$. We have

$$
\sum_{n=0}^{\infty} \frac{f(n)}{n+\alpha}=\frac{-1}{q} \sum_{a=0}^{q-1} f(a) \psi\left(\frac{a+\alpha}{q}\right) .
$$

Proof. For $\operatorname{Re}(s)>1$,

$$
\begin{aligned}
\sum_{n=0}^{\infty} \frac{f(n)}{(n+\alpha)^{s}} & =\frac{1}{q^{s}} \sum_{a=0}^{q-1} f(a) \sum_{m=0}^{\infty} \frac{1}{(m+(a+\alpha) / q)^{s}} \\
& =\frac{1}{q^{s}} \sum_{a=0}^{q-1} f(a) \zeta\left(s, \frac{a+\alpha}{q}\right) .
\end{aligned}
$$

This last series appears to have a simple pole at $s=1$ since the Hurwitz zeta function has such a pole. Luckily, the residue at $s=1$ is $\sum_{a=0}^{q-1} f(a) / q=0$, which is both necessary and sufficient for convergence. Thus, we have analytic continuation of our series via analytic continuation of the Hurwitz zeta function. By the same convergence condition, we insert $\sum_{a=0}^{q-1} f(a) \zeta(s)=0$ into our sum, which gives

$$
\frac{1}{q^{s}} \sum_{a=0}^{q-1} f(a)\left(\zeta\left(s, \frac{a+\alpha}{q}\right)-\zeta(s)\right) .
$$

Note that

$$
\lim _{s \rightarrow 1^{+}} \zeta(s, x)-\zeta(s)=\frac{1}{x}+\sum_{n=1}^{\infty}\left(\frac{1}{n+x}-\frac{1}{n}\right),
$$

which equals $-\psi(x)-\gamma$ by (2). Thus, taking the limit as $s$ goes to 1 , we have

$$
\sum_{n=0}^{\infty} \frac{f(n)}{n+\alpha}=\frac{-1}{q} \sum_{a=0}^{q-1} f(a) \psi\left(\frac{a+\alpha}{q}\right) .
$$

We can now say something about the transcendence of such a series by incorporating Baker's theorem after using equation (3) to relate the series to a linear form in logarithms. Instead of stating a theorem for the previous series, we prove a more general result for $\sum_{n=0}^{\infty} f(n) A(n) / B(n)$. For now we assume that the roots of $B(x)$ are in the short interval [-1,0). In [1], the authors call $B(x)$ reduced if all of its coefficients are rational and it 
has simple rational roots in this interval, but we do not require as many assumptions. Later we will relax this restriction on the roots altogether.

Corollary 5. Let $A(x), B(x) \in \overline{\mathbb{Q}}[x]$ be such that $\operatorname{deg}(A)<\operatorname{deg}(B)$ and $B(x)$ has simple roots in $[-1,0)$. Let $f$ be an algebraic-valued periodic function as above, with $\sum_{a=0}^{q-1} f(a)=0$. The series

$$
\sum_{n=0}^{\infty} \frac{f(n) A(n)}{B(n)}
$$

is either zero or transcendental.

Proof. Let $-\alpha_{1}, \ldots,-\alpha_{k}$ be the roots of $B(x)$, so that each $\alpha_{i} \in(0,1]$. By partial fractions we write

$$
\frac{A(x)}{B(x)}=\sum_{i=1}^{k} \frac{c_{i}}{x+\alpha_{i}}
$$

where each $c_{i}$ is algebraic. We see that our series equals

$$
\sum_{i=1}^{k} c_{i} \sum_{n=0}^{\infty} \frac{f(n)}{n+\alpha_{i}}
$$

which in turn equals

$$
\frac{-1}{q} \sum_{i=1}^{k} c_{i} \sum_{a=0}^{q-1} f(a)\left(\psi\left(\frac{a+\alpha_{i}}{q}\right)+\gamma\right)
$$

by Theorem 4. Write $\alpha_{i}=p_{i} / q_{i}$ and by (3) we obtain

$$
\frac{-1}{q} \sum_{i=1}^{k} c_{i} \sum_{a=0}^{q-1} f(a)\left(-\log \left(q_{i} q\right)+\sum_{b=1}^{q_{i} q-1} \zeta_{q_{i} q}^{-b\left(a q_{i}+p_{i}\right)} \log \left(1-\zeta_{q_{i} q}^{b}\right)\right),
$$

which is equal to

$$
-\sum_{i=1}^{k} c_{i} \sum_{b=1}^{q_{i} q-1} \widehat{f}(b) \zeta_{q_{i} q}^{-b p_{i}} \log \left(1-\zeta_{q_{i} q}^{b}\right)
$$

where $\widehat{f}(b)=(1 / q) \sum_{a=0}^{q-1} f(a) \zeta_{q}^{-a b}$ is the discrete Fourier transform. We have an algebraic linear combination of logarithms of algebraic numbers. By Baker's theorem the sum is either zero or transcendental.

At this point it seems impossible to characterize exactly when the series is equal to zero. One reason is that for various integers $q_{i} q$, as in the proof above, the values $\log \left(1-\zeta_{q_{i} q}^{b}\right)$ are not linearly independent over $\mathbb{Q}$. Thus, in order to check whether or not the series is zero, it is not as simple as checking a single coefficient for a given log term. The coefficients of each log term could be zero in some nontrivial way or there could be some linear 
dependence. Without further assumption, nothing can be concluded at this point.

The convergence condition $\sum_{a=0}^{q-1} f(a)=0$ does not allow us to characterize all types of series of this form. We next change our setting ever so slightly relaxing the convergence condition placed on $f$, and instead require that $\operatorname{deg}(A)<\operatorname{deg}(B)-1$ to ensure that we have convergence. Although this case does have overlap with the previous case, it is worth examining both cases for completeness. They are of course strikingly similar.

THEOREM 6. Let $f$ be an algebraic-valued periodic function modulo $q \geq 1$. Let $A(x), B(x) \in \overline{\mathbb{Q}}[x]$ be such that $\operatorname{deg}(A)<\operatorname{deg}(B)-1$ and $B(x)$ has simple roots in $[-1,0)$. The series

$$
\sum_{n=0}^{\infty} \frac{f(n) A(n)}{B(n)}
$$

is either zero or transcendental.

Proof. Let $-\alpha_{1}, \ldots,-\alpha_{k}$ be the roots of $B(x)$. Since $B(x)$ has only simple roots, by partial fractions we can write

$$
\frac{A(x)}{B(x)}=\sum_{i=1}^{k} \frac{c_{i}}{x+\alpha_{i}}
$$

where each $c_{i}$ is an algebraic number. It turns out that $c_{i}=A\left(-\alpha_{i}\right) / B^{\prime}\left(-\alpha_{i}\right)$ and considering the degrees of $A$ and $B$ we see that

$$
\sum_{i=1}^{k} c_{i}=0 .
$$

For reasons that will soon become clear, we separate our sum into two parts:

$$
\sum_{n=q}^{\infty} \frac{f(n) A(n)}{B(n)}+\sum_{a=0}^{q-1} \frac{f(a) A(a)}{B(a)},
$$

and examine the first of these two sums. We have

$$
\sum_{n=q}^{\infty} \frac{f(n) A(n)}{B(n)}=\sum_{a=0}^{q-1} f(a) \sum_{n \equiv a(q), n \geq q} \sum_{i=1}^{k} \frac{c_{i}}{n+\alpha_{i}} .
$$

Since $\sum_{i=1}^{k} c_{i}=0$, we can insert $\sum_{i=1}^{k}-c_{i} /(n-a)$ into the innermost sum and obtain

$$
\sum_{a=0}^{q-1} f(a) \sum_{n \equiv a(q), n \geq q} \sum_{i=1}^{k} c_{i}\left(\frac{1}{n+\alpha_{i}}-\frac{1}{n-a}\right),
$$


which equals

$$
\frac{1}{q} \sum_{a=0}^{q-1} f(a) \sum_{i=1}^{k} c_{i} \sum_{m=1}^{\infty}\left(\frac{1}{m+\left(a+\alpha_{i}\right) / q}-\frac{1}{m}\right) .
$$

By (2), the innermost sum is equal to $-\psi\left(\left(a+\alpha_{i}\right) / q\right)-\gamma-q /\left(a+\alpha_{i}\right)$ and this last sum is equal to

$$
\frac{-1}{q} \sum_{a=0}^{q-1} f(a) \sum_{i=1}^{k} c_{i}\left(\psi\left(\frac{a+\alpha_{i}}{q}\right)+\gamma\right)-\sum_{a=0}^{q-1} \frac{f(a) A(a)}{B(a)} .
$$

Notice that the last sum here is exactly the part which we omitted earlier. Putting it all together we have

$$
\sum_{n=0}^{\infty} \frac{f(n) A(n)}{B(n)}=\frac{-1}{q} \sum_{a=0}^{q-1} f(a) \sum_{i=1}^{k} c_{i}\left(\psi\left(\frac{a+\alpha_{i}}{q}\right)+\gamma\right) .
$$

Similar to the proof of Corollary 5 we utilize equation (3) to relate values of the digamma function to a linear combination of logarithms. Writing $\alpha_{i}=p_{i} / q_{i}$, by $(3)$, we obtain

$$
\frac{-1}{q} \sum_{a=0}^{q-1} f(a) \sum_{i=1}^{k} c_{i}\left(-\log \left(q_{i} q\right)+\sum_{b=1}^{q_{i} q-1} \zeta_{q_{i} q}^{-b\left(a q_{i}+p_{i}\right)} \log \left(1-\zeta_{q_{i} q}^{b}\right)\right) .
$$

At this point we simplify by inserting the discrete Fourier transform of $f$ to obtain

$$
\sum_{i=1}^{k} c_{i}\left(\widehat{f}(0) \log \left(q_{i} q\right)-\sum_{b=1}^{q_{i} q-1} \widehat{f}(b) \zeta_{q_{i} q}^{-b p_{i}} \log \left(1-\zeta_{q_{i} q}^{b}\right)\right) .
$$

The end result is again a linear form in logarithms of algebraic numbers. By Baker's theorem, we conclude that the series $\sum_{n=0}^{\infty} f(n) A(n) / B(n)$ is either zero or transcendental.

Note that in Corollary 5 we assumed that $\widehat{f}(0)=(1 / q) \sum_{a=0}^{q-1} f(a)=0$. Thus, the formula shown in Theorem 4 reduces to the same form found in Corollary 5. Thus, both cases coincide.

Ideally, we would like to know exactly when series of these types converge to zero. However, as stated above, it is difficult to characterize when they vanish. For now, we leave that problem open and move onto a more general series. We relax the restriction that the roots of $B$ all lie in $[-1,0)$. Obviously, this could cause problems if $B(x)$ has a root in the positive integers, thus we simply take summation over all values of $n \geq 0$ where $B(n) \neq 0$. The end result is very similar, with the exception that instead of obtaining a linear form in logarithms of algebraic numbers, we obtain an algebraic number plus a linear form in logarithms. We show this result here. 
THEOREM 7. Let $f$ be an algebraic-valued periodic function with integer period $q \geq 1$. Let $A(x), B(x) \in \overline{\mathbb{Q}}[x]$ be such that $B(x)$ has only simple roots in $\mathbb{Q}$. Omitting the roots of $B(x)$, the series

$$
\sum_{n=0}^{\infty} \frac{f(n) A(n)}{B(n)}
$$

is equal to a computable algebraic number or is transcendental, when it converges.

Proof. Let $-\alpha_{1}, \ldots,-\alpha_{k}$ be the roots of $B(x)$. Take the minimal natural number $r=q t$ such that $\left|\alpha_{i}\right| \leq r-1$. We separate our series into two parts:

$$
\sum_{n=0}^{r-1} \frac{f(n) A(n)}{B(n)}+\sum_{n=r}^{\infty} \frac{f(n) A(n)}{B(n)}=\beta_{1}+\sum_{n=0}^{\infty} \frac{f(n+r) A(n+r)}{B(n+r)} .
$$

By partial fractions

$$
\frac{A(x+r)}{B(x+r)}=\sum_{i=1}^{k} \frac{c_{i}}{x+r+\alpha_{i}}
$$

and by similar methods to the above we relate our series to the digamma function and obtain

$$
\beta_{1}+\frac{-1}{q} \sum_{i=1}^{k} c_{i} \sum_{a=0}^{q-1} f(a)\left(\psi\left(\frac{a+r+\alpha_{i}}{q}\right)+\gamma\right) .
$$

For each pair $(a, i)$ we shift via equation (1) to write

$$
\psi\left(\frac{a+r+\alpha_{i}}{q}\right)=\psi\left(\frac{p_{i, a}}{q_{i, a}}\right)+\beta_{i, a}
$$

where $p_{i, a} / q_{i, a} \in(0,1]$ and $\beta_{i, a}$ are rational numbers. Note that if we write $\alpha_{i}=p_{i} / q_{i}$, then $q_{i, a}=q_{i} q$. This allows us to once again use equation (3) and we see that our sum is equal to

$$
\beta+\frac{1}{q} \sum_{i=1}^{k} c_{i} \sum_{a=0}^{q-1} f(a)\left(\log \left(q_{i} q\right)-\sum_{b=1}^{q_{i} q-1} \zeta_{q_{i} q}^{-b p_{i, a}} \log \left(1-\zeta_{q_{i} q}^{b}\right)\right)
$$

where $\beta=\beta_{1}-(1 / q) \sum_{i=1}^{k} c_{i} \sum_{a=0}^{q-1} f(a) \beta_{i, a}$. Notice $q=\prod_{b=1}^{q-1}\left(1-\zeta_{q}^{b}\right)$ so $\log q=\sum_{b=1}^{q-1} \log \left(1-\zeta_{q}^{b}\right)$. Using this last equality we simplify our sum to

$$
\beta-\frac{1}{q} \sum_{i=1}^{k} c_{i} \sum_{a=0}^{q-1} f(a) \sum_{b=1}^{q_{i} q-1}\left(\zeta_{q_{i} q}^{-b p_{i, a}}-1\right) \log \left(1-\zeta_{q_{i} q}^{b}\right) .
$$

Our series is equal to a finite sum of algebraic numbers, depending only on $f, A$ and $B$, plus an algebraic linear combination of logarithms of algebraic numbers. By Baker's theorem we are done. 
Clearly, it is not easy to characterize exactly when such a series is algebraic. However, due to a formula of Gauss and a lemma of Murty and Saradha [9] we can give conditions that will guarantee that our series is transcendental.

We have the following formula of Gauss which can be used to give an alternative closed form for the types of series we are interested in. Gauss first showed the formula in 1813, but Lehmer has a nice proof in [7]. For $1 \leq a<q$,

(5) $\psi(a / q)+\gamma=-\log 2 q-\frac{\pi}{2} \cot \frac{\pi a}{q}+2 \sum_{0<j \leq q / 2}\left(\cos \frac{2 \pi a j}{q}\right) \log \sin \frac{\pi j}{q}$.

Gauss's formula relates the digamma function and $\gamma$ to a sum of logarithms of positive algebraic numbers plus an algebraic multiple of $\pi$. The $\pi$ that appears is important due to the following lemma, which we state without proof.

Lemma 8 (Murty/Saradha, [9]). Let $\alpha_{1}, \ldots, \alpha_{n}$ be positive algebraic numbers. If $c_{0}, c_{1}, \ldots, c_{n}$ are algebraic numbers with $c_{0} \neq 0$, then

$$
c_{0} \pi+\sum_{j=1}^{n} c_{j} \log \alpha_{j} \neq 0
$$

and is a transcendental number.

Applying this lemma to the series in Theorem 7 we have the following theorem.

Theorem 9. Let $A(x), B(x)$ and $f$ be as in Theorem 7. For each pair $(a, i)$, take $s_{a, i} \equiv a q_{i}+p_{i}\left(\bmod q q_{i}\right)$ to be the unique integer such that $0<$ $s_{a, i} \leq q q_{i}$. With summation taken over only those terms with $s_{a, i} \neq q q_{i}$, if

$$
\sum_{i=1}^{k} c_{i} \sum_{a=0}^{q-1} f(a) \cot \left(\frac{\pi s_{a, i}}{q q_{i}}\right) \neq 0
$$

then the series

$$
\sum_{n=0}^{\infty} \frac{f(n) A(n)}{B(n)}
$$

is transcendental.

Proof. Similar to the proof of Theorem 7, our series is equal to

$$
\beta_{1}-\frac{1}{q} \sum_{i=1}^{k} c_{i} \sum_{a=0}^{q-1} f(a)\left(\psi\left(\frac{a+r+\alpha_{i}}{q}\right)+\gamma\right) .
$$

We shift each digamma value the appropriate amount using equation (1) to 
obtain an algebraic number plus

$$
-\frac{1}{q} \sum_{i=1}^{k} c_{i} \sum_{a=0}^{q-1} f(a)\left(\psi\left(\frac{s_{a, i}}{q q_{i}}\right)+\gamma\right) .
$$

Since $\psi(1)+\gamma=0$, we omit those terms with $s_{a, i}=q q_{i}$. After substitution via Gauss's formula (5) for $\psi\left(s_{a, i} / q q_{i}\right)+\gamma$, we collect terms to obtain the coefficient of $\pi$ and obtain the result by Lemma 8 .

We now have a simple criterion to check whether a given series is transcendental. Unfortunately, the condition given in Theorem 9 is sufficient but not necessary for transcendence. The following theorem illustrates at least one general case when a series of this form is equal to a transcendental number.

Theorem 10. Let $A(x), B(x) \in \overline{\mathbb{Q}}[x]$ with $\operatorname{deg}(A)<\operatorname{deg}(B)-1$ be such that $B(x)$ has only simple roots $-p_{1} / q_{1}, \ldots,-p_{k} / q_{k} \in \mathbb{Q}$ with $\left(p_{i}, q_{i}\right)=1$. If there is a $q_{j}>1$ which is coprime to each of the other $q_{i}$ 's, then the sum

$$
\sum_{n=0}^{\infty} \frac{A(n)}{B(n)}
$$

is transcendental.

Proof. Without loss of generality, we assume that $q_{1}>1$ and $\left(q_{1}, q_{i}\right)=1$ for each $i \neq 1$. Similar to the proof of Theorem 7 , let $r$ be the minimal natural number $r=q t$ such that $r>\left|p_{i} / q_{i}\right|$ for every $i=1, \ldots, k$ so that

$$
\sum_{n=0}^{\infty} \frac{A(n)}{B(n)}=\beta-\sum_{i=1}^{k} c_{i}\left(\psi\left(r+p_{i} / q_{i}\right)+\gamma\right)
$$

for some $\beta \in \overline{\mathbb{Q}}$. Equation (1) allows us to rewrite our sum as

$$
\widetilde{\beta}-\sum_{i=1}^{k} c_{i}\left(\psi\left(\widetilde{p_{i}} / q_{i}\right)+\gamma\right),
$$

where $0<\widetilde{p_{i}} \leq q_{i}$ and $\widetilde{\beta} \in \overline{\mathbb{Q}}$.

Recall from Theorem 3 that $\psi\left(\widetilde{p_{1}} / q_{1}\right)+\gamma$ is transcendental, and therefore not equal to zero. By (3), we have

$$
\widetilde{\beta}-\sum_{i=1}^{k} c_{i}\left(-\log q_{i}+\sum_{b=1}^{q_{i}-1} \zeta_{q_{i}}^{-b \widetilde{p}_{i}} \log \left(1-\zeta_{q_{i}}^{b}\right)\right),
$$

which equals

$$
\widetilde{\beta}-\sum_{i=1}^{k} c_{i} \sum_{b=1}^{q_{i}-1}\left(\zeta_{q_{i}}^{-b \widetilde{p}_{i}}-1\right) \log \left(1-\zeta_{q_{i}}^{b}\right) .
$$


We wish to show that the logarithmic part of the summation does not vanish, and conclude the result via Baker's theorem.

Let $T_{q_{1}} \subseteq\left\{\log \left(1-\zeta_{q_{1}}\right), \ldots, \log \left(1-\zeta_{q_{1}}^{q_{1}-1}\right)\right\}$ be a maximal set of linearly independent (over $\mathbb{Q}$ ) terms. Let $S=\left\{q_{i}: q_{i}>1, i=2, \ldots, k\right\}$. If $S$ is empty we are finished, so suppose $S$ is not empty. Take $T_{S} \subseteq\left\{\log \left(1-\zeta_{q_{i}}^{j}\right): q_{i} \in S\right.$, $\left.j=1, \ldots, q_{i}-1\right\}$ be any maximal linearly independent set. A nontrivial linear relation between $T_{q_{1}}$ and $T_{S}$ can be written as

$$
x_{1} \log \left(1-\zeta_{q_{1}}^{a_{1}}\right)+\cdots+x_{s} \log \left(1-\zeta_{q_{1}}^{a_{s}}\right)=\sum_{q_{i} \in S} \sum_{j=1}^{t_{i}} y_{i, j} \log \left(1-\zeta_{q_{i}}^{b_{i, j}}\right)
$$

for some integers (not all zero) $x_{i}, a_{i}, t_{i}, y_{i, j}$ and $b_{i, j}$. This implies that

$$
\prod_{i=1}^{s}\left(1-\zeta_{q_{1}}^{a_{i}}\right)^{x_{i}}=\prod_{q_{i} \in S} \prod_{j=1}^{t_{i}}\left(1-\zeta_{q_{i}}^{b_{i, j}}\right)^{y_{i, j}} .
$$

We recall some facts from algebraic number theory. The ring of integers of any cyclotomic extension, $\mathbb{Q}\left(\zeta_{m}\right)$, is equal to $\mathbb{Z}\left[\zeta_{m}\right]$ ([8, Exercise 4.5.25]). Thus, since $\prod_{i=1}^{m-1}\left(1-\zeta_{m}^{i}\right)=m$, the norm (in any field extension over $\left.\mathbb{Q}\left(\zeta_{m}\right)\right)$ of any factor $1-\zeta_{m}^{i}$ is an integer dividing some power of $m$. With this in mind, we take the norm $N_{K}$ of both sides of equation (6) where $K=\mathbb{Q}\left(\zeta_{q_{1}}, \ldots, \zeta_{q_{k}}\right)$. Since $\left(q_{1}, q_{i}\right)=1$ for every $i=2, \ldots, k$, we have

$$
N_{K}\left(\prod_{i=1}^{s}\left(1-\zeta_{q_{1}}^{a_{i}}\right)^{x_{i}}\right)= \pm 1=N_{K}\left(\prod_{q_{i} \in S} \prod_{j=1}^{t_{i}}\left(1-\zeta_{q_{i}}^{b_{i, j}}\right)^{y_{i, j}}\right) .
$$

Since $\mathbb{Q}\left(\zeta_{q_{1}}\right) \cap \mathbb{Q}\left(\zeta_{q_{2}}, \ldots, \zeta_{q_{k}}\right)=\mathbb{Q}$, the two products in $(6)$ are in fact \pm 1 . Squaring equation (6) and then taking the log of both sides, we have

$$
x_{1} \log \left(1-\zeta_{q_{1}}^{a_{1}}\right)+\cdots+x_{s} \log \left(1-\zeta_{q_{1}}^{a_{s}}\right)=0=\sum_{q_{i} \in S} \sum_{j=1}^{t_{i}} y_{i, j} \log \left(1-\zeta_{q_{i}}^{b_{i, j}}\right) .
$$

This is a contradiction, thus $T_{q_{1}} \cup T_{S}$ is a set of linearly independent values over $\mathbb{Q}$. Theorem 3 shows that the part of the sum involving the terms $\log \left(1-\zeta_{q_{1}}^{b}\right)$ for $b=1, \ldots, q_{1}-1$ does not vanish, therefore the series is transcendental.

4. Infinite sums and polygamma functions. We next relax the restriction that $B(x)$ has only simple roots. When $B(x)$ has simple roots, we obtain a linear form in special values of the digamma function. The next lemma shows a connection between the multiplicity of roots of $B(x)$ and special values of the polygamma function.

LEMMA 11. Let $f$ be a periodic function with integer period $q$. Take $k \geq 1$ and $\alpha \in \mathbb{R} \backslash \mathbb{Z}_{\leq 0}$. Then 


$$
\sum_{n \geq 0} \frac{f(n)}{(n+\alpha)^{k}}=\frac{(-1)^{k}}{q^{k}(k-1) !} \sum_{a=1}^{q} f(a) \psi_{k-1}\left(\frac{a+\alpha}{q}\right)
$$

when the series converges.

Proof. The case $k=1$ is in the previous section, so assume that $k \geq 2$. We have

$$
\sum_{n \geq 0} \frac{f(n)}{(n+\alpha)^{k}}=\frac{1}{q^{k}} \sum_{a=0}^{q-1} f(a) \sum_{n \geq 0} \frac{1}{(n+(a+\alpha) / q)^{k}},
$$

which, by 4 , equals

$$
\frac{(-1)^{k}}{q^{k}(k-1) !} \sum_{a=0}^{q-1} f(a) \psi_{k-1}\left(\frac{a+\alpha}{q}\right) .
$$

We are now able to calculate closed forms for series with very general $B(x)$.

THEOREM 12. Let $f$ be an algebraic-valued periodic function, with period $q \geq 1$. Take any $A(x), B(x) \in \overline{\mathbb{Q}}[x]$ with $-\alpha_{1}, \ldots,-\alpha_{k} \in \mathbb{Q} \backslash \mathbb{Z}_{\geq 0}$ the distinct roots of $B(x)$ with multiplicities $m_{1}, \ldots, m_{k}$ respectively. If the series converges, then

$$
\sum_{n \geq 0} \frac{f(n) A(n)}{B(n)}
$$

is an algebraic linear combination of values of various polygamma functions at rational points.

Proof. For the series to converge, we must have at least $\operatorname{deg}(A)<\operatorname{deg}(B)$ and so, by partial fractions, we can write

$$
\frac{A(x)}{B(x)}=\sum_{i=1}^{k} \sum_{j=1}^{m_{i}} \frac{c_{i, j}}{\left(x+\alpha_{i}\right)^{j}}
$$

Inserting this into our series we have

$$
\sum_{n \geq 0} \frac{f(n) A(n)}{B(n)}=\sum_{i=1}^{k} \sum_{j=1}^{m_{i}} c_{i, j} \sum_{n \geq 0} \frac{f(n)}{\left(n+\alpha_{i}\right)^{j}},
$$

which equals

$$
\sum_{i=1}^{k} \sum_{j=1}^{m_{i}} \frac{(-1)^{j} c_{i, j}}{q^{j}(j-1) !} \sum_{a=0}^{q-1} f(n) \psi_{j-1}\left(\frac{a+\alpha_{i}}{q}\right)
$$

by the previous lemma.

Note that we could use the same trick which was demonstrated in the proof of Theorem [7. We could shift the summation to exclude all of the 
zeroes of $B(x)$, which allows us to include the case where $B(x)$ has any rational zero. This method was demonstrated earlier, and we simply state the result without proof.

THEOREM 13. Let $f$ be an algebraic-valued periodic function, with period $q \geq 1$. Take any $A(x), B(x) \in \overline{\mathbb{Q}}[x]$. With the zeroes of $B(x)$ omitted when necessary, if the series converges then

$$
\sum_{n=0}^{\infty} \frac{f(n) A(n)}{B(n)}
$$

is a computable algebraic number plus an algebraic linear combination of values of various polygamma functions at rational points.

5. Exponential polynomial sums. For this section we remove the periodic function and insert an exponential. That is, we are interested in series of the form

$$
\sum_{n=0}^{\infty} \frac{z^{n} A(n)}{B(n)}
$$

where $A(x), B(x)$ are as before, and $z \in \overline{\mathbb{Q}}$. We begin with a simple case, where $A(x)$ is constant and $B(x)$ is a single linear polynomial with rational root. That is, for $\alpha \in \mathbb{Q} \backslash \mathbb{Z}$, we examine

$$
\sum_{n=0}^{\infty} \frac{z^{n}}{n+\alpha}
$$

which converges on $|z| \leq 1$ with $z \neq 1$. The following lemma will play an important role in our analysis.

Lemma 14. For $p / q \in(0,1]$ with $(p, q)=1$ and any $|z| \leq 1, z \neq 0,1$, we have

$$
\sum_{n=0}^{\infty} \frac{z^{n}}{n+p / q}=-z^{-p / q} \sum_{t=0}^{q-1} \zeta_{q}^{-p t} \log \left(1-\zeta_{q}^{t} z^{1 / q}\right) .
$$

Proof. For now, assume that $|z|<1$. We have

$$
-\sum_{t=0}^{q-1} \zeta_{q}^{-p t} \log \left(1-\zeta_{q}^{t} z^{1 / q}\right)=\sum_{t=0}^{q-1} \zeta_{q}^{-p t} \sum_{n=1}^{\infty} \frac{\left(\zeta_{q}^{t} z^{1 / q}\right)^{n}}{n},
$$

which equals

$$
z^{p / q} \sum_{m=0}^{\infty} \frac{z^{m}}{m+p / q} .
$$

Thus we have the result for $|z|<1$. We extend equality via Abel's convergence theorem which extends equality of a function and its power series inside the unit circle to the boundary, as long as the series converges. 
In the case where $p / q$ is not in $(0,1]$, we simply shift our summation so that we have a finite sum of terms plus an infinite series similar to that which we analyze in Lemma 14. We demonstrate this in the following transcendence result.

Proposition 15. Let $z \neq 1$ be an algebraic number with $|z| \leq 1$. For any $p / q \in \mathbb{Q}$ with $(p, q)=1$, the series

$$
\sum_{n=0}^{\infty} \frac{z^{n}}{n+p / q}
$$

is a computable algebraic number or is transcendental.

Proof. If $z=0$ we are done, so assume that $z \neq 0$. Write $p / q=r+\widetilde{p} / \widetilde{q}$ where $\widetilde{p} / \widetilde{q} \in(0,1]$. Rewrite the series as

$$
z^{-r} \sum_{n=0}^{\infty} \frac{z^{n+r}}{n+r+\widetilde{p} / \widetilde{q}}
$$

which equals

$$
S+z^{-r} \sum_{n=0}^{\infty} \frac{z^{n}}{n+\widetilde{p} / \widetilde{q}}
$$

where

$$
S= \begin{cases}-z^{-r} \sum_{n=0}^{r-1} \frac{z^{n}}{n+\widetilde{p} / \widetilde{q}} & \text { if } r>0 \\ z^{-r} \sum_{n=r}^{-1} \frac{z^{n}}{n+\widetilde{p} / \widetilde{q}} & \text { if } r<0 .\end{cases}
$$

Note that $\sum^{\prime}$ here is summation avoiding the original $-p / q$, if needed. If $r=0$ then no shift is needed and $S=0$. With $S$ explicitly given, the remainder of the sum is determined by Lemma 14 . Our series is equal to

$$
S-z^{-r-\widetilde{p} / \widetilde{q}} \sum_{t=0}^{\widetilde{q}-1} \zeta_{\widetilde{q}}^{\widetilde{p} t} \log \left(1-\zeta_{\widetilde{q}}^{t} z^{1 / \widetilde{q}}\right),
$$

which simplifies to

$$
S-z^{-p / q} \sum_{t=0}^{q-1} \zeta_{q}^{-p t} \log \left(1-\zeta_{q}^{t} z^{1 / q}\right)
$$

By Baker's theorem we are done.

We next analyze the case when we have an exponential with a polynomial. Though it is easy to see that in this case the series equals an algebraic 
number, we compute the closed form relating the series to Stirling numbers of the second kind.

Proposition 16. For $P(x) \in \overline{\mathbb{Q}}[x]$ and $z$ algebraic with $|z|<1$, the series

$$
\sum_{n=0}^{\infty} z^{n} P(n)
$$

is algebraic.

Proof. Assume that $z \neq 0$, or else we are done. It is easy to see that the series converges absolutely, thus for $P(x)=\sum_{i=0}^{k} a_{i} x^{i}$ we write

$$
\sum_{n=0}^{\infty} z^{n} P(n)=\sum_{i=0}^{k} a_{i} \sum_{n=0}^{\infty} z^{n} n^{i} .
$$

Note that

$$
x^{i}=\sum_{j=0}^{i} S(i, j)(x)_{j}
$$

where $(x)_{j}$ is the falling factorial defined as $x(x-1) \cdots(x-j+1)$ for $j \geq 1$, $(x)_{0}=1$, and $S(n, k) \in \mathbb{N}$ is a Stirling number of the second kind. Inserting this into our series we have

$$
\sum_{i=0}^{k} a_{i} \sum_{j=0}^{i} S(i, j) \sum_{n=0}^{\infty}(n)_{j} z^{n}
$$

which equals

$$
\sum_{i=0}^{k} a_{i} \sum_{j=0}^{i} S(i, j) z^{j} \sum_{n=0}^{\infty}(n+1) \cdots(n+j) z^{n} .
$$

The innermost sum is precisely the $j$ th derivative of a well-known series,

$$
\left(\frac{z^{j}}{1-z}\right)^{(j)}=\frac{j !}{(1-z)^{j+1}}
$$

and we have

$$
\sum_{n=0}^{\infty} z^{n} P(n)=\sum_{i=0}^{k} a_{i} \sum_{j=0}^{i} \frac{S(i, j) j ! z^{j}}{(1-z)^{j+1}},
$$

which is an algebraic number if $z$ is algebraic.

From Propositions 15 and 16 we immediately have the following theorem. 
TheOREM 17. Let $z \neq 1$ be algebraic with $|z| \leq 1$. Let $A(x), B(x) \in \overline{\mathbb{Q}}[x]$ be such that $B(x)$ has simple rational roots. If it converges, the series

$$
\sum_{n=0}^{\infty} \frac{z^{n} A(n)}{B(n)}
$$

is a computable algebraic number or is transcendental.

Proof. Write $A(x) / B(x)=Q(x)+R(x) / B(x)$ where $Q(x), R(x) \in \overline{\mathbb{Q}}[x]$ and $\operatorname{deg}(R)<\operatorname{deg}(B)$. Assuming convergence, we have either $|z|<1$, or $|z|=1(z \neq 1)$ and $Q(x)=0$. By partial fractions we write

$$
\frac{R(x)}{B(x)}=\sum_{i=1}^{k} \frac{c_{i}}{x+p_{i} / q_{i}}
$$

so that

$$
\sum_{n=0}^{\infty} \frac{z^{n} A(n)}{B(n)}=\sum_{n=0}^{\infty} z^{n} Q(n)+\sum_{i=1}^{k} c_{i} \sum_{n=0}^{\infty} \frac{z^{n}}{n+p_{i} / q_{i}}
$$

where each infinite sum omits the roots of $B(x)$. We conclude the result by Propositions 15 and 16 .

Note that Theorem 17 encompasses Theorem 7. Recall that by Fourier inversion on a finite group, $f(n)$ can be written

$$
f(n)=\widehat{f}(0)+\widehat{f}(1) \zeta_{q}^{n}+\cdots+\widehat{f}(q-1) \zeta_{q}^{n(q-1)} .
$$

We then can write

$$
\begin{aligned}
\sum_{n=0}^{\infty} \frac{f(n) A(n)}{B(n)} & =\sum_{n=0}^{\infty} \frac{A(n)}{B(n)} \sum_{a=0}^{q-1} \widehat{f}(a) \zeta_{q}^{a n} \\
& =\sum_{a=0}^{q-1} \widehat{f}(a) \sum_{n=0}^{\prime} \frac{\left(\zeta_{q}^{a}\right)^{n} A(n)}{B(n)}
\end{aligned}
$$

and by Theorem 17 we immediately have Theorem 7 as a corollary.

For the sake of completeness, we mention one final series with a general setting of exponentials with polynomials. The theorem is Theorem 4 of [1]. With the theory developed here it is possible to prove the general result, however, this author has no improvement on the proof of the authors of [1]. We state the theorem, but refer the reader to [1] for the complete proof. Note that there is a small mistake in the proof of Theorem 4 from [1]. The authors relate their series to a sum of a computable algebraic number and a linear form in logarithms of algebraic numbers. In the second last line of their proof, the authors simply forget the computable algebraic number. 
With the statement of their theorem fixed, as given here, we can also drop a condition that was imposed on $Q(x)$. The condition that $Q(x)$ have all of its roots in $[-1,0)$ is no longer needed. With the edited statement, the theorem is true with $Q(x)$ having any simple rational roots.

Theorem 18 (Theorem 4 of [1]). Let $P_{1}(x), \ldots, P_{l}(x) \in \overline{\mathbb{Q}}[x]$ and $\alpha_{1}, \ldots$, $\alpha_{l} \in \overline{\mathbb{Q}}$. Put $g(x)=\sum_{i=1}^{l} \alpha_{i}^{x} P_{i}(x)$. Let $Q(x) \in \overline{\mathbb{Q}}[x]$ have simple rational roots. If the series

$$
\sum_{n=0}^{\infty} \frac{g(n)}{Q(n)}
$$

converges, then the sum is either a computable algebraic number or transcendental.

Also note that with the theory developed in this section, we could simply add various convergent series and obtain a result similar to the statement of this theorem. It turns out, as shown in [1, that the only series of this general form which converge are those which can be formed by adding convergent series of the type discussed in Theorem 17.

We remark that some results found here are similar to those found in [1], but the methods used here are different. Our methods were inspired by [10].

Acknowledgments. This research was supported by an NSERC-CGS award.

\section{References}

[1] S. D. Adhikari, N. Saradha, T. N. Shorey, and R. Tijdeman, Transcendental infinite sums, Indag. Math. (N.S.) 12 (2001), 1-14.

[2] A. Baker, Transcendental Number Theory, 2nd ed., Cambridge Math. Lib., Cambridge Univ. Press, Cambridge, 1990, 165 pp.

[3] A. Baker, B. Birch, and E. Wirsing, On a problem of Chowla, J. Number Theory 5 (1973), 224-236.

[4] P. Bundschuh, Zwei Bemerkungen über transzendente Zahlen, Monatsh. Math. 88 (1979), 293-304.

[5] S. Chowla, The nonexistence of nontrivial linear relations between the roots of a certain irreducible equation, J. Number Theory 2 (1970), 120-123.

[6] Kh. Hessami Pilehrood and T. Hessami Pilehrood, Infinite sums as linear combinations of polygamma functions, Acta Arith. 130 (2007), 231-254.

[7] D. H. Lehmer, Euler constants for arithmetical progressions, ibid. 27 (1975), 125142 .

[8] M. R. Murty and J. Esmonde, Problems in Algebraic Number Theory, 2nd ed., Grad. Texts in Math. 190, Springer, New York, 2005, 352 pp.

[9] M. R. Murty and N. Saradha, Euler-Lehmer constants and a conjecture of Erdős, J. Number Theory, to appear. 
[10] M. R. Murty and N. Saradha, Transcendental values of the digamma function, ibid. 125 (2007), 298-318.

Chester Weatherby

Department of Mathematics and Statistics

Queen's University

Kingston, Ontario, K7L 3N6, Canada

E-mail: cjwthrb@mast.queensu.ca 